\title{
Strain Induced Electronic and Optical Properties of 2D Silicon Carbide Monolayer Using Density Functional Theory
}

\author{
S. Bhandari Sharma, R. Adhikari, K. R. Sigdel and R.Bhatta
}

Journal of Nepal Physical Society

Volume 7, Issue 1, April 2021

(Special Issue: ANPA Conference, 2020)

ISSN: 2392-473X (Print), 2738-9537(Online)

Editors:

Dr. Santosh KC

San Jose State University, USA (Editor in Chief)

Dr. Pashupati Dhakal

Thomas Jefferson National Accelerator Facility, USA

Dr. Yadav Pandit

Baptist Health Science University, USA

Managing Editor:

Dr. Binod Adhikari

St. Xavier's College, Kathmandu, Nepal

JNPS, 7 (1), 60-65 (2021)

DOI: http://doi.org/10.3126/jnphyssoc.v7i1.36977

Published by:

Nepal Physical Society

P.O. Box: 2934

Tri-Chandra Campus

Kathmandu, Nepal

Email: npseditor@gmail.com

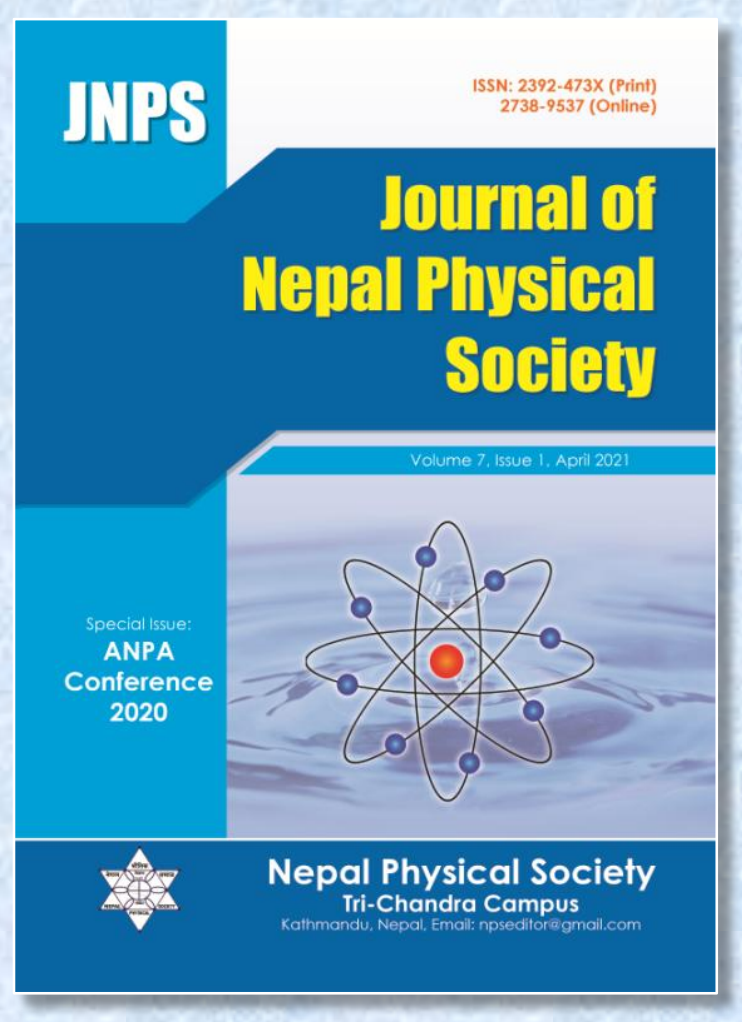




\title{
Strain Induced Electronic and Optical Properties of 2D Silicon Carbide Monolayer Using Density Functional Theory
}

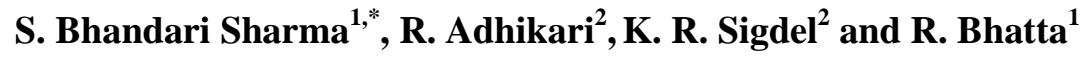 \\ ${ }^{1}$ Goldengate College, Tribhuvan University, Kathmandu, Nepal \\ ${ }^{2}$ Kathmandu University Kavre, Nepal \\ "Corresponding Email: shambhubhandari789@gmail.com
}

Received: 10 August, 2020; Revised: 9 March, 2021; Accepted: 11 March, 2021

\begin{abstract}
Using the first principles calculation, we investigated the structural, electronic, and straindependent optical properties of the two-dimensional hexagonal Silicon Carbide (SiC) Monolayer. We found that the biaxial compressive strain loading gradually changes the direct bandgap SiC into indirect bandgap semiconductor. The compressive strain increases the bandgap but reduces the values of static dielectric constant and refractive index. Conversely, the biaxial tensile strain loading decreases the bandgap but increases the value of static dielectric constant and refractive index. The result shows that the electronic and optical properties of $\mathrm{SiC}$ can be engineered to the desired value by applying strain. The large bandgap issue for the $\mathrm{SiC}$ monolayer is limiting its uses in different applications which can be overcome with the help of biaxial strain.
\end{abstract}

Keywords: DFT; Strain; Optical properties.

\section{INTRODUCTION}

After the discovery of graphene [1], twodimensional graphene-like materials have drawn considerable attention from many researchers. The hexagonal graphene-like Silicon Carbide $(\mathrm{SiC})$ is an energetically, mechanically, and chemically stable [2] two-dimensional material. It exhibits a wide direct bandgap. This semiconducting property allows it in fabricating the light-emitting diodes (LEDs), Solar cells, and many other optoelectronic device applications. Monolayer SiC shows better photoluminescence (PL) than its bulk counterpart. This can be illustrated by the quantum confinement effect. Compared to other semiconductors $(\mathrm{GaN}$, $\mathrm{BP}, \mathrm{AlN}$, etc.) monolayer $\mathrm{SiC}$ has the largest value of in-plane stiffness [3]. Recent studies show that the local magnetic moment in the $\mathrm{SiC}$ monolayer appears only in the presence of silicon-vacancy in the monolayer [4]. Recent progress in the fabrication of $\mathrm{SiC}$ monolayer shows that it is an excellent semiconductor for different optoelectronic device applications.

In this work, we comparatively studied the electronic and optical properties of the $\mathrm{SiC}$ monolayer at compressive and tensile strain loading regimes using the density functional theory.

\section{COMPUTATIONAL DETAILS}

All the calculations presented in this paper are done using the density functional theory as implemented in the Spanish Initiative for Electronic Simulations with Thousands of Atoms(SIESTA) code [5,6]. The pseudopotential is constructed using Troullier Martins Method [7]. The generalized gradient approximation (GGA) [8]function with PerdewBurke-Ernzerhof(PBE) [8] is adopted to treat the effects of correlation and electronic exchange. In all the calculations, we used the double zeta plus polarization (DZP) as basis sets. Monkhrost pack [9] scheme has been used for Brillouin zone integration using $15 \times 15 \times 1$.An energy cutoff of 450 Ry for the expansion of the reciprocal space. The atomic relaxation was achieved when the force reached the value of $0.002 \mathrm{eV} / \AA$ using the standard conjugate-gradient (CG) technique. The convergence criteria for the energy of the selfconsistent field is set to be $1.0 \times 10^{-5} \AA$. The vacuum gap of $25 \AA$ between adjacent direction has 
been used to prevent interactions between the adjacent unit cells.

Optical properties are calculated by applying the electromagnetic field in parallel to the $2 d$ monolayer plane. Optical spectra are made smooth by applying Gaussian broadening value to $0.02 \mathrm{eV}$. The optical mesh points are chosen to be $45 \times 45 \times 3$. The $f$-sum rule [10] was obtained within the energy range of $0-40 \mathrm{eV}$. The self-consistent ground state energies and Eigenfunction is used in the dipolar transition matrix, to calculate the imaginary part of the dielectric function $\left(\varepsilon_{2}\right)$, the real part $\left(\varepsilon_{1}\right)$ of the dielectric function, reflectance spectra, absorption coefficient refractive index are investigated. For inplane biaxial $\operatorname{strain}\left(\varepsilon=\left(a-a_{0}\right) / a_{0}\right)$, is applied equally in XY-plane, were $a_{0}$ and $a$ are the lattice constants of the system before and after the deformation, respectively. The strain $(\varepsilon)$ is loaded by varying the lattice vectors from $-16 \%$ to $+16 \%$ and then electronic and optical properties are analyzed.

(a)

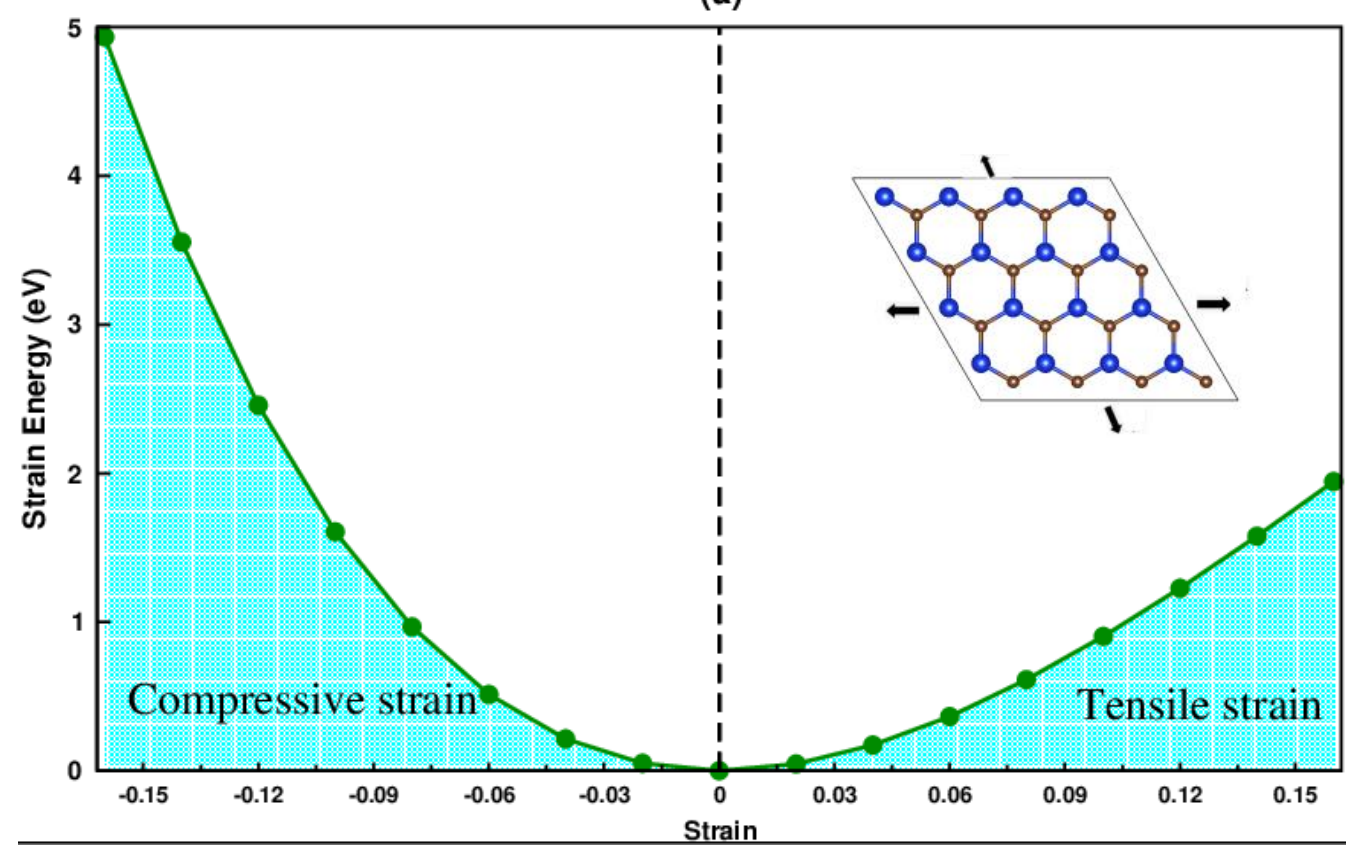

Fig. 1. Biaxial strain loading regime for $\mathrm{SiC}$ monolayer around the harmonic elastic range

\section{RESULTS AND DISCUSSION}

\subsection{Strain effect on the structural and electronic properties of $\mathrm{SiC}$ monolayer.}

The monolayer structure optimized without spin effect has lattice constant and bond distance between silicon and carbon $3.10 \AA$ and $1.80 \AA$ respectively. The cohesive energy of the monolayer is calculated by subtracting the energy of isolated atoms of $\mathrm{Si}$ and $\mathrm{C}$ from the compound silicon carbide total energy. The cohesive energy of the monolayer is $-6.81 \mathrm{eV} /$ atom indicates a highly stable compound. Further biaxial strain $(\varepsilon)$ is loaded with increasing compressive and strain within the elastic range. The minimum strain energy is located at equilibrium structure $(0 \%$ strain) indicating that the most stable structure of monolayer $\mathrm{SiC}$ is the strain free-structure. The lattice parameters ( $a=b)$ and bond is increasing linearly with the strain increment. The strain-free monolayer is a direct bandgap semiconductor with a bandgap of $2.32 \mathrm{eV}$. The band structure alongside DOS is depicted in Fig. 2. The calculated structural and electronic properties fairly agrees with the previously reported results [11]. Although, the GGA-PBE calculation underestimates the bandgap we try to explore the change in the electronic band structure of the monolayer by applying the biaxial strain shown in Fig. 2. The bandgap is increasing during compressive strain and decreases during tensile strain as illustrated in figure 3 . The change in bandgap is due to the (i) repulsive effect between valence and conduction band [12], (ii) delocalization effect [13]. 

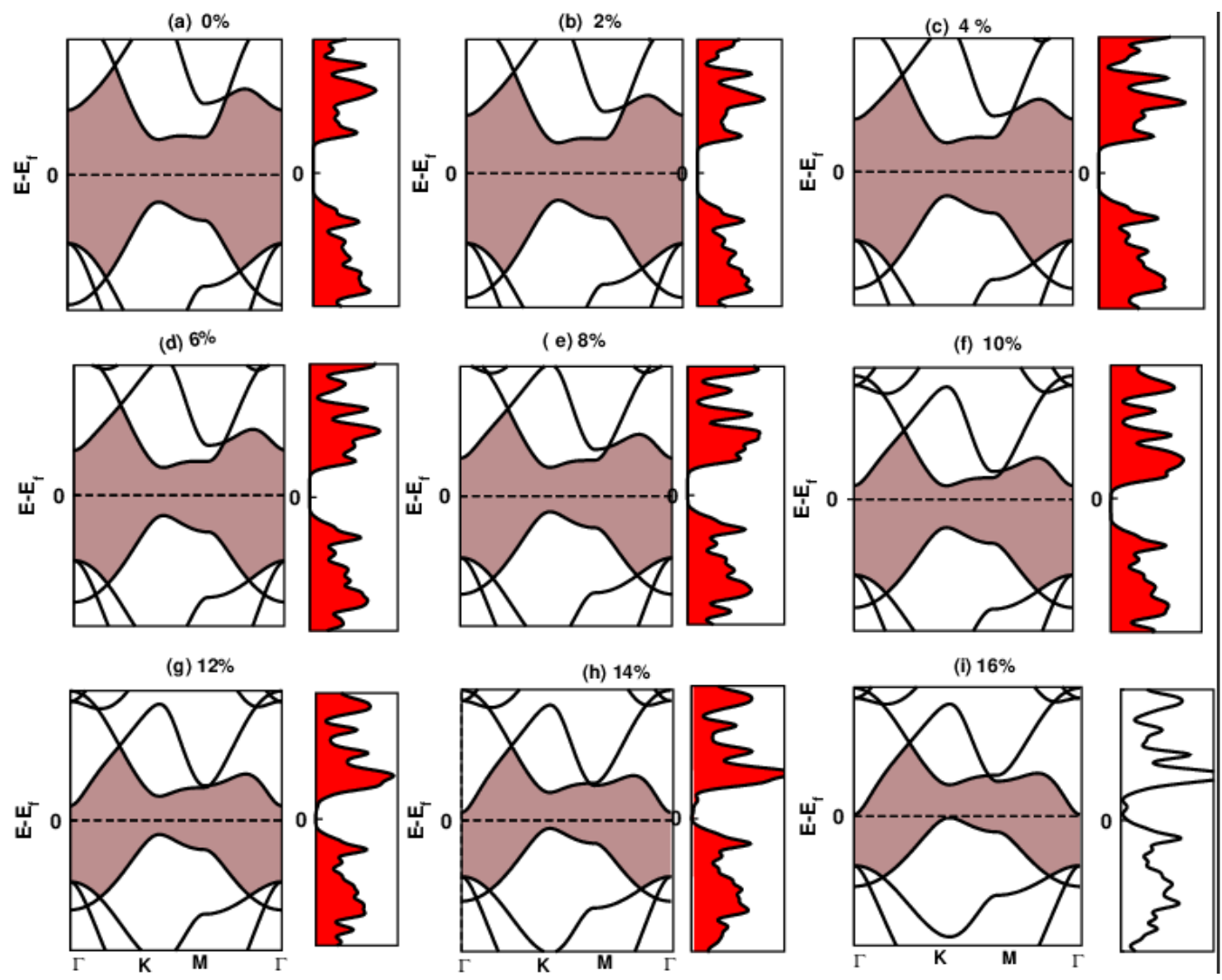

Fig. 2: (a) Electronic band structure alongside DOS of the SiC monolayer at a different value of biaxial tensile strain, the horizontal line lines show the Fermi level.

\subsection{Strain effect on the optical properties of $\mathrm{SiC}$ monolayer}

In optical properties, we calculate the imaginary and real part of the dielectric function, static dielectric constant, refractive index, and absorption coefficient for the parallel incidence of light on the monolayer for the compressive strain $\left(a_{i}\right)$ and

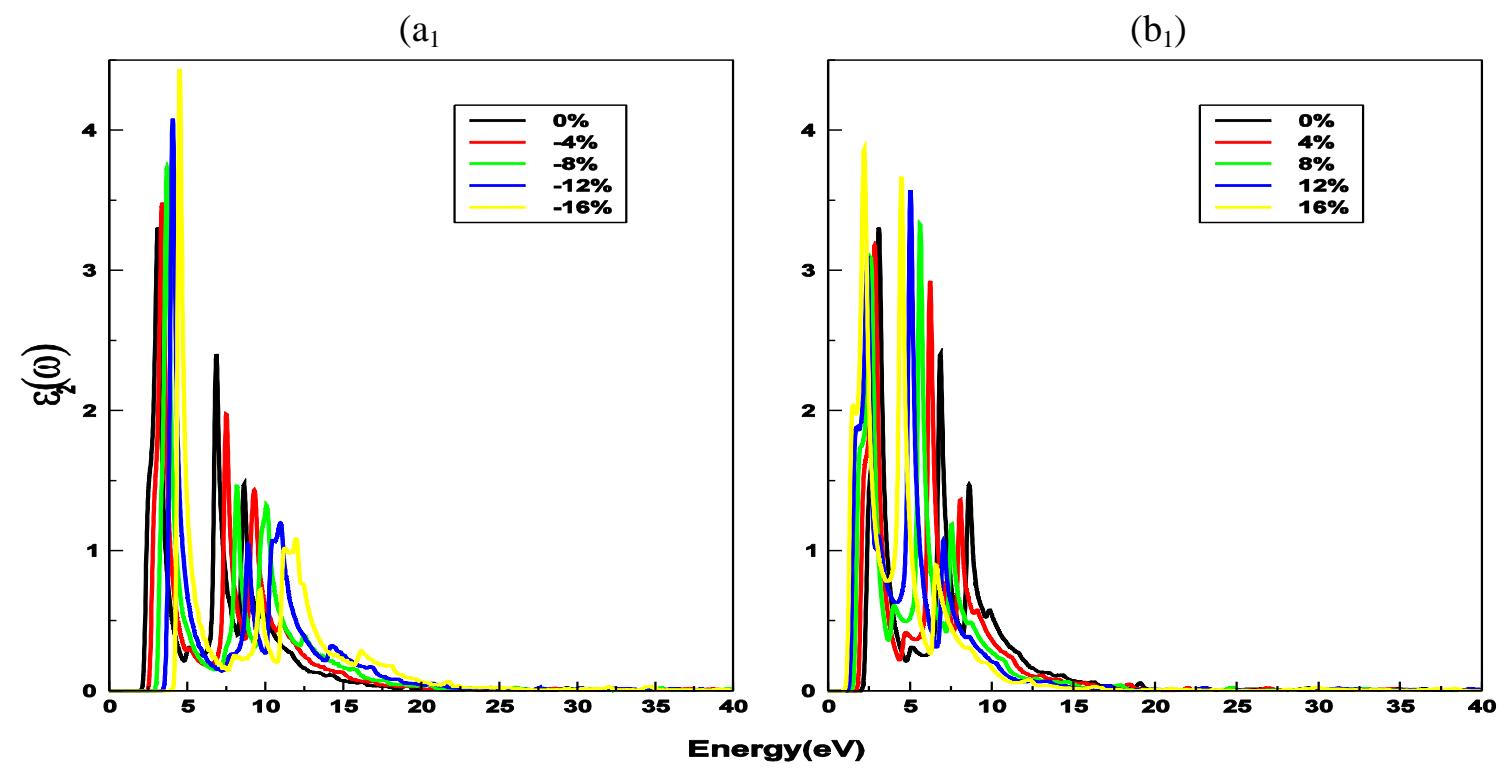

tensile strain $\left(b_{i}\right)$ is shown in Fig. 3. Optical spectra for the compressive strain are decreased in compressive strain whereas, increased for tensile strain. The static dielectric constant, static refractive index value increases for tensile strain and decreases for the compressive strain.

$\left(b_{1}\right)$ 
$\left(a_{2}\right)$

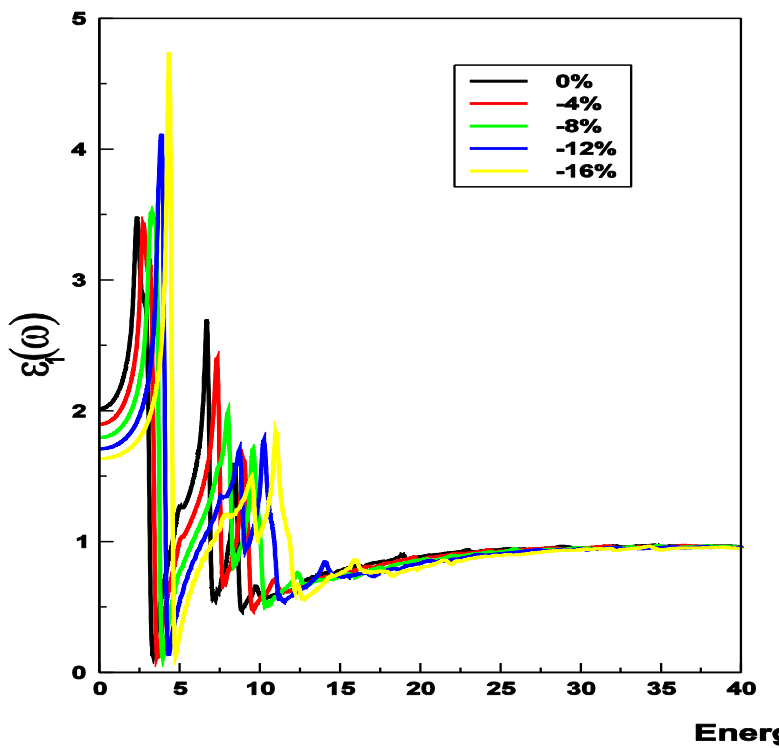

$\left(a_{3}\right)$

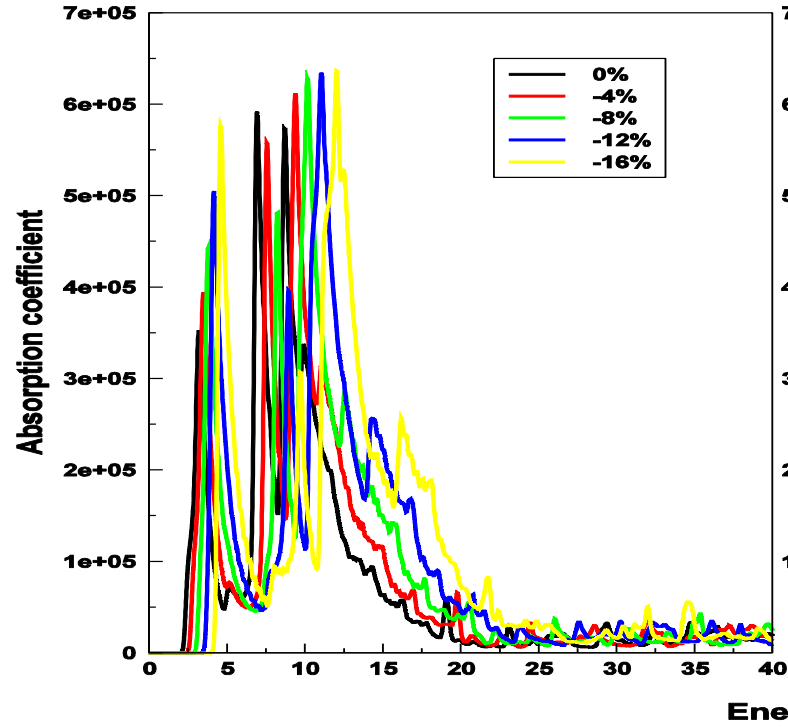

$\left(\mathrm{a}_{4}\right)$

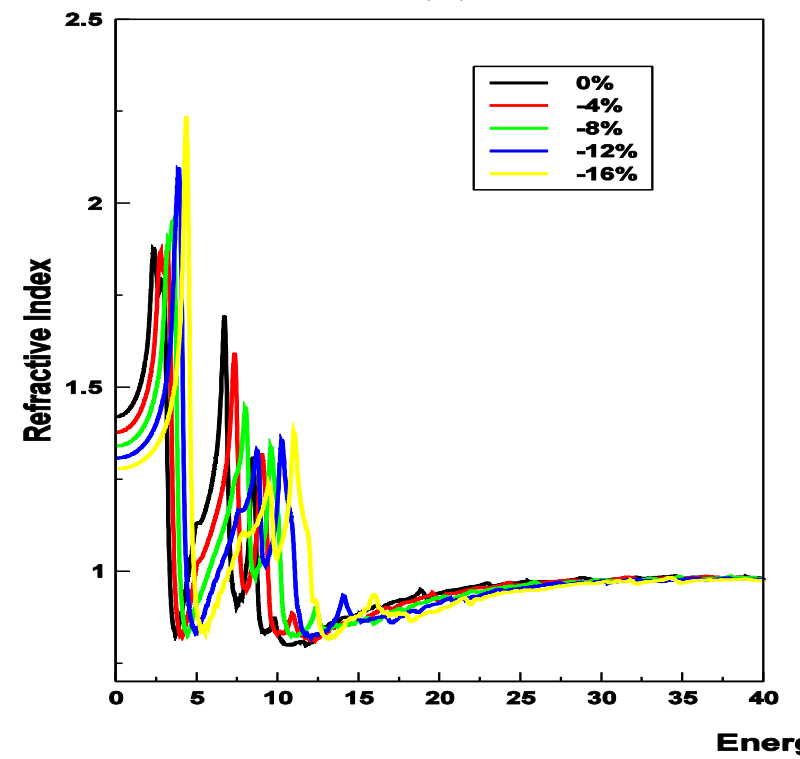

$\left(b_{2}\right)$

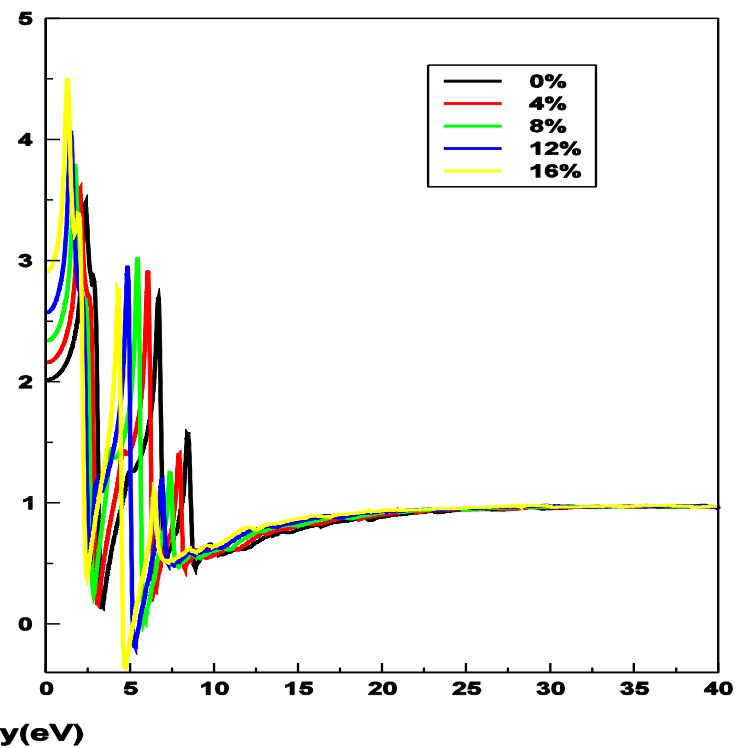

$\left(b_{3}\right)$

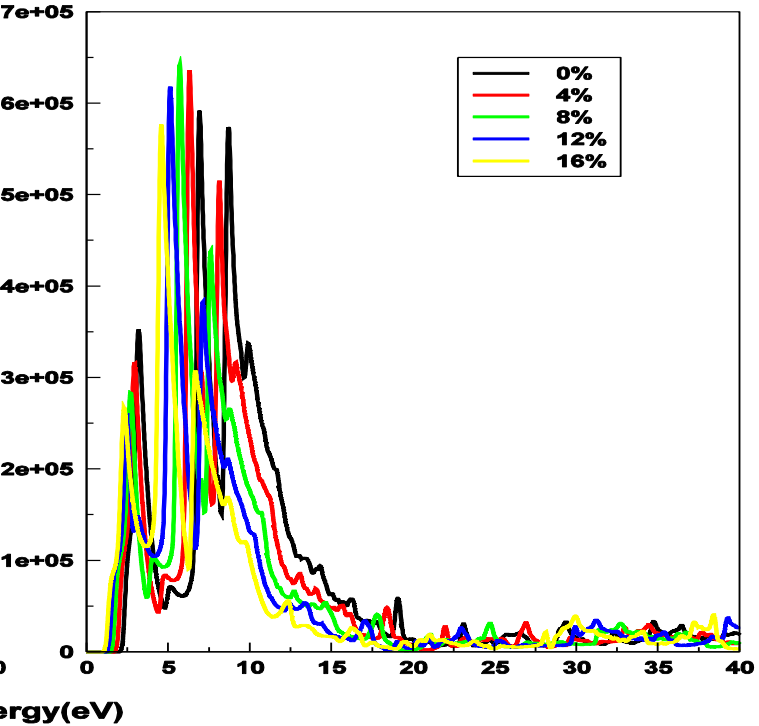

$\left(\mathrm{b}_{4}\right)$

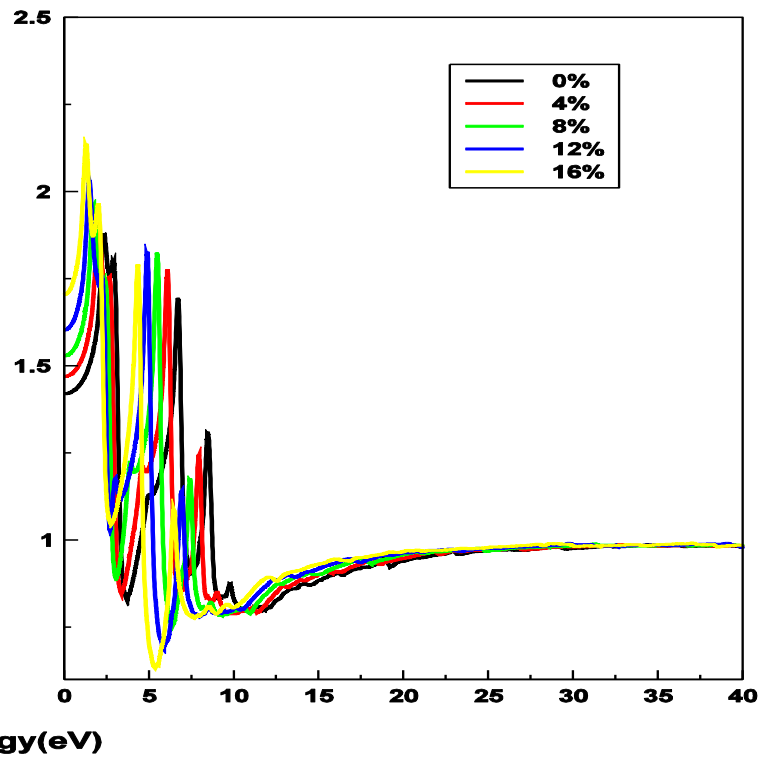


$\left(\mathrm{a}_{5}\right)$

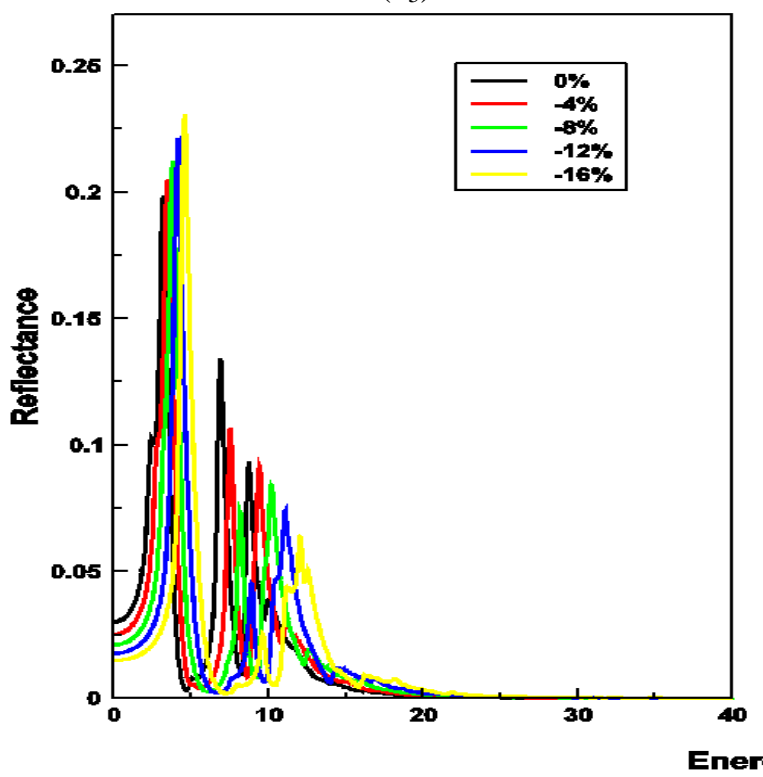

$\left(b_{5}\right)$

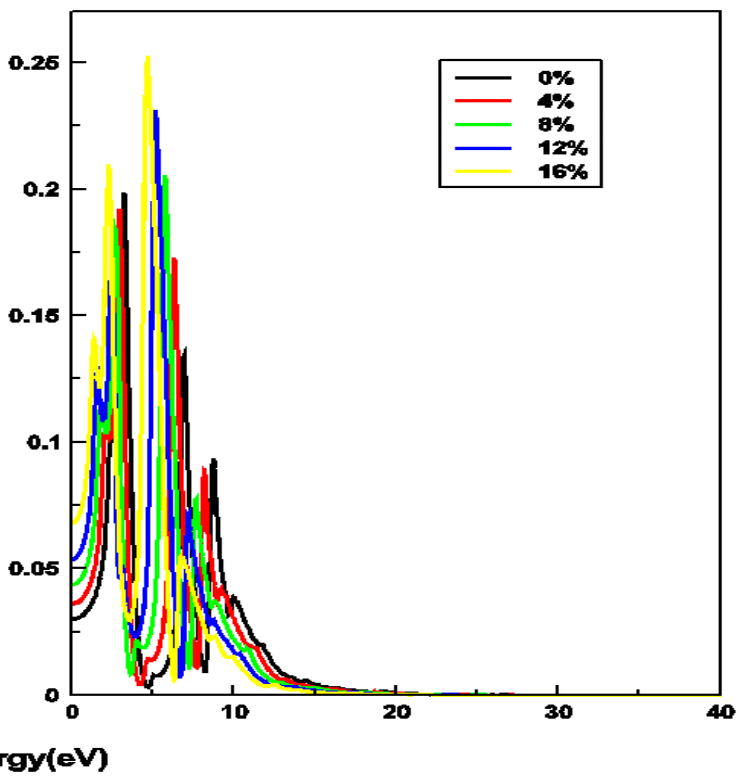

Fig. 3: Optical parameters: $\left(a_{1}, b_{1}\right)$ imaginary dielectric function $\left(a_{2}, b_{2}\right)$ real part of dielectric function $\left(a_{3}, b_{3}\right)$ absorption coefficient $\left(a_{4}, b_{4}\right)$ refractive index $\left(a_{5}, b_{5}\right)$ reflectance.

The value of static dielectric constant $\left(\epsilon_{0}\right)$ is the value of the dielectric function $\left(\epsilon_{1}\right)$ at zero energy and static refractive index $\left(\mu_{0}\right)$ is the value of the refraction index at zero energy. The value of static dielectric constant and refractive index at strainfree case is found to be $2.12 \mathrm{eV}, 1.42$ respectively. This result is in good agreement with the previously reported data [14]. The values of $\epsilon_{0}$ increases continuously with tensile strain and decrease by the compressive strain. A similar result is observed in the case of static refractive index as shown in Fig.3. Moreover, the reflection peaks show that the monolayer is almost transparent above $20 \mathrm{eV}$ for strain-free as well as strain loading conditions. The highest absorption peaks for monolayer occurs at $8.1 \mathrm{eV}$ of photon energy for the strain-free condition which gradually varies with the applied strain.

\section{CONCLUSIONS}

The effect of strain on electronic and optical properties of $\mathrm{SiC}$ is investigated using Density Functional Theory. The bandgap increases during compressive strain and decreases for tensile strain. Also, optical properties can be altered by applying strain which can be implemented in next-generation optoelectronic devices.

\section{ACKNOWLEDGEMENTS}

This research was supported in part through computational resources provided by the Kathmandu University Supercomputer Centre, which was established with equipment donated by CERN.

\section{CONFLICTS OF INTEREST}

The authors declare no conflict of interest.

\section{EDITOR'S NOTE}

This manuscript was submitted to Association of Nepali Physicists in America (ANPA) Conference 2020 for publication in special issue of Journal of Nepal Physical Society.

\section{REFERENCES}

[1] Novoselov, K. S.; Geim, A. K.; Morozov, S. V.; Jiang, D.; Zhang, Y.; Dubonos, S. V.; Grigorieva, I. V. and Firsov, A. A. Electric Field in Atomically Thin Carbon Films, Science (80-...) (2004).

[2] Bekaroglu, E.; Topsakal, M.; Cahangirov, S. and Ciraci, S. First-Principles Study of Defects and Adatoms in Silicon Carbide Honeycomb Structures, Phys. Rev. B - Condens. Matter Mater. Phys. (2010).

[3] Şahin, H.; Cahangirov, S.; Topsakal, M.; 
Bekaroglu, E.; Akturk, E.; Senger, R. T. and Ciraci, S. Monolayer Honeycomb Structures of Group-IV Elements and III-V Binary Compounds: First-Principles Calculations, Phys. Rev. B Condens. Matter Mater. Phys. 80: 1 (2009).

[4] Eliseeva, N. S.; Kuzubov, A. A.; Ovchinnikov, S. G.; Serzhantova, M. V.; Tomilin, F. N. and Fedorov, A. S. Theoretical Study of the Magnetic Properties of Ordered Vacancies in $2 D$ Hexagonal Structures: Graphene, 2D-SiC, and h$B N$, JETP Lett. (2012).

[5] Soler, J. M.; Artacho, E.; Gale, J. D.; García, A.; Junquera, J.; Ordejón, P. and Sánchez-Portal, D. The SIESTA Method for Ab Initio Order-N Materials Simulation, J. Phys. Condens. Matter 14: 2745 (2002).

[6] Ordejón, P.; Artacho, E. and Soler, J. M. SelfConsistent Order Density-Functional Calculations for Very Large Systems, Phys. Rev. B - Condens. Matter Mater. Phys. 53: R10441 (1996).

[7] Troullier N. and Martins, J. L. Efficient Pseudopotentials for Plane-Wave Calculations, Phys. Rev. B (1991).

[8] Perdew, J. P.; Burke, K. and Ernzerhof, M. Generalized Gradient Approximation Made Simple, Phys. Rev. Lett. (1996).

[9] Monkhorst, H. J. and Pack, J. D. Special Points for Brillouin-Zone Integrations, Phys. Rev. B (1976).

[10] Kemp, B. A. Resolution of the AbrahamMinkowski Debate: Implications for the Electromagnetic Wave Theory of Light in Matter, Journal of Applied Physics.

[11] Lin, X. ; Lin, S.; Xu, Y.; Hakro, A. A.; Hasan, T.; Zhang, B.; Yu, B.; Luo, J.; Li, E. and Chen, H. Ab Initio Study of Electronic and Optical Behavior of Two-Dimensional Silicon Carbide, J. Mater. Chem. C (2013).

[12] Wei, S. H. and Zunger, A. Predicted Band-Gap Pressure Coefficients of All Diamond and ZincBlende Semiconductors: Chemical Trends, Phys. Rev. B - Condens. Matter Mater. Phys. (1999).

[13] Lü, T. Y.; Liao, X. X.; Wang, H. Q. and Zheng, J. C. Tuning the Indirect-Direct Band Gap Transition of SiC, GeC and SnC Monolayer in a Graphene-like Honeycomb Structure by Strain Engineering: A Quasiparticle GW Study, J. Mater. Chem. 22: 10062 (2012).

[14] Majidi, S.; Elahi, S. M.; Esmailian, A. and Kanjouri, F. First Principle Study of Electronic and Optical Properties of Planar GeC, SnC and SiC Nanosheets, Prot. Met. Phys. Chem. Surfaces 53: 773 (2017). 